\title{
DYNAMICS OF A SECOND-ORDER SYSTEM OF NONLINEAR DIFFERENCE EQUATIONS
}

\author{
ERKAN TAŞDEMIR
}

Kırklareli University,

Pınarhisar Vocational School of Higher Education, 39300, Kırklareli, Turkey erkantasdemir@hotmail.com, erkan.tasdemir@klu.edu.tr

\section{Abstract}

In this paper, we investigate the equilibrium points, stability of two equilibrium points, convergences of negative equilibrium point, periodic solutions, and existence of bounded or unbounded solutions of a system of nonlinear difference equations

$$
x_{n+1}=x_{n-1} y_{n}-1, y_{n+1}=y_{n-1} x_{n}-1
$$

$n=0,1, \ldots$, where the initial values are real numbers. Additionally we present some numerical examples to verify our theoretical results.

2010 AMS Classification: 39A10, 39A30, 39A23, 39A24

Keywords: Difference equation, stability, global stability, periodicity, eventually periodicity

\section{INTRODUCTION}

During the last years, difference equations or discrete dynamical systems are a fertile research area, not only in mathematics but also in many applied science such as economics, genetics. Moreover, difference equations or discrete dynamic systems apply to different fields of science as mathematical models. From this, many scientists, mathematicians or not, have frequently studied this topic. In particular, these researchers are interested in the stability of solutions, periodic solutions, and bounded or unbounded solutions. There are many papers to dynamical systems for example,

In [3], Camouzis et al studied global behaviour of solutions of the system of difference equations

$$
x_{n+1}=1+\frac{x_{n}}{y_{n-m}}, y_{n+1}=1+\frac{y_{n}}{x_{n-m}} .
$$

In [25], Stevic studied the system of difference equations

$$
x_{n+1}=\frac{u_{n}}{1+v_{n}}, y_{n+1}=\frac{w_{n}}{1+s_{n}}
$$

where $u_{n}, v_{n}, w_{n}, s_{n}$ are some subsequences $x_{n}$ or $y_{n}$.

In [17], Kurbanlı et al studied behaviour of positive solutions of system of difference equations

$$
x_{n+1}=\frac{x_{n-1}}{y_{n} x_{n-1}+1}, y_{n+1}=\frac{y_{n-1}}{x_{n} y_{n-1}+1} .
$$


In [10], Kent et al studied long-term behaviour of solutions of difference equation

$$
x_{n+1}=x_{n} x_{n-1}-1 .
$$

Further, in [30], Wang et al and in [18], Liu et al obtained some significant results about related difference equation. Furthermore, there are many books and papers related to difference equations see [1] - [30].

In this study, we investigate the dynamics of following the system of difference equations

$$
x_{n+1}=x_{n-1} y_{n}-1, y_{n+1}=y_{n-1} x_{n}-1, n=0,1, \ldots,
$$

where all initial values are real numbers. Especially, we study equilibrium points, stability of solutions, existence of periodic solutions and bounded or unbounded solutions of related system of difference equations.

Now, we present some definitions and theorems which are used throughout this study.

Let us introduce a four-dimensional discrete dynamical system of the form

$$
x_{n+1}=f\left(x_{n}, x_{n-1}, y_{n}, y_{n-1}\right), y_{n+1}=g\left(x_{n}, x_{n-1}, y_{n}, y_{n-1}\right),
$$

$n=0,1, \ldots$, where $f: I^{4} \times J^{4} \rightarrow I$ and $g: I^{4} \times J^{4} \rightarrow J$ are continuously differentiable functions and $I, J$ are some intervals of real numbers. Moreover, a solution $\left\{\left(x_{n}, y_{n}\right)\right\}_{n=-1}^{\infty}$ of system (1.2) is uniquely determined by initial values $\left(x_{i}, y_{i}\right) \in I \times J$ for $i \in\{-1,0\}$.

Definition 1. Along with the system (1.2), we consider the corresponding vector map $F=\left\{f, x_{n}, x_{n-1}, g, y_{n}, y_{n-1}\right\}$. A point $(\bar{x}, \bar{y})$ is called an equilibrium point of the system (1.2) if

$$
\bar{x}=f(\bar{x}, \bar{x}, \bar{y}, \bar{y}), \bar{y}=g(\bar{x}, \bar{x}, \bar{y}, \bar{y}) .
$$

The point $(\bar{x}, \bar{y})$ is also called a fixed point of the vector map $F$.

Definition 2. Let $(\bar{x}, \bar{y})$ be an equilibrium point of the system (1.2).

(i): An equilibrium point $(\bar{x}, \bar{y})$ of system (1.2) is called stable if, for every $\varepsilon>0$, there exists $\delta>0$ such that, for every initial value $\left(x_{-i}, y_{-i}\right) \in I \times J$, with

$$
\sum_{i=-1}^{0}\left|x_{i}-\bar{x}\right|<\delta, \quad \sum_{i=-1}^{0}\left|y_{i}-\bar{y}\right|<\delta,
$$

implying $\left|x_{n}-\bar{x}\right|<\varepsilon$ and $\left|y_{n}-\bar{y}\right|<\varepsilon$ for $n \in \mathbb{N}$.

(ii): An equilibrium point $(\bar{x}, \bar{y})$ of system (1.2) is called unstable, if it is not stable.

(iii): An equilibrium point $(\bar{x}, \bar{y})$ of system (1.2) is called locally asymptotically stable if it is stable and if, in addition, there exists $\gamma>0$ such that

$$
\sum_{i=-1}^{0}\left|x_{i}-\bar{x}\right|<\gamma, \quad \sum_{i=-1}^{0}\left|y_{i}-\bar{y}\right|<\gamma
$$

and $\left(x_{n}, y_{n}\right) \rightarrow(\bar{x}, \bar{y})$ as $n \rightarrow \infty$.

(iv): An equilibrium point $(\bar{x}, \bar{y})$ of system (1.2) is called a global attractor if $\left(x_{n}, y_{n}\right) \rightarrow(\bar{x}, \bar{y})$ as $n \rightarrow \infty$.

(v): An equilibrium point $(\bar{x}, \bar{y})$ of system (1.2) is called globally asymptotically stable if it is stable and a global attractor. 
Definition 3. Let $(\bar{x}, \bar{y})$ be an equilibrium point of the map $F$ where $f$ and $g$ are continuously differentiable functions at $(\bar{x}, \bar{y})$. The linearized system of system (1.2) about the equilibrium point $(\bar{x}, \bar{y})$ is

$$
X_{n+1}=F\left(X_{n}\right)=B X_{n}
$$

where

$$
X_{n}=\left(\begin{array}{c}
x_{n} \\
x_{n-1} \\
y_{n} \\
y_{n-1}
\end{array}\right)
$$

and $B$ is a Jacobian matrix of system (1.2) about the equilibrium point $(\bar{x}, \bar{y})$.

Definition 4. Assume that $X_{n+1}=F\left(X_{n}\right), n=0,1, \cdots$, is a system of difference equations such that $\bar{X}$ is a fixed point of $F$. If no eigenvalues of the Jacobian matrix $B$ about $\bar{X}$ have absolute value equal to one, then $\bar{X}$ is called hyperbolic. Otherwise, $\bar{X}$ is said to be nonhyperbolic.

Theorem 1 (Linearized Stability Theorem [14], p.11). Assume that

$$
X_{n+1}=F\left(X_{n}\right), n=0,1, \cdots,
$$

is a system of difference equations such that $\bar{X}$ is a fixed point of $F$.

(i): If all eigenvalues of the Jacobian matrix $B$ about $\bar{X}$ lie inside the open unit disk $|\lambda|<1$, that is, if all of them have absolute value less than one, then $\bar{X}$ is locally asymptotically stable.

(ii): If at least one of them has a modulus greater than one, then $\bar{X}$ is unstable.

Definition 5. A solution $\left\{\left(x_{n}, y_{n}\right)\right\}_{n=-1}^{\infty}$ of system (1.2) is bounded and persists if there exist constants $M, N$ such that $M<N$ and

$$
M<x_{n}, y_{n}<N, n=-m,-m+1, \cdots
$$

Definition 6. A positive solution $\left\{\left(x_{n}, y_{n}\right)\right\}_{n=-1}^{\infty}$ of system (1.2) is periodic with period $p$ if

$$
x_{n+p}=x_{n}, y_{n+p}=y_{p} \text { for all } n \geq-1 .
$$

\section{Equilibrium Points of System (1.1)}

In this here, we study equilibrium points of system (1.1).

Theorem 2. System (1.1) has two equilibrium points which are

$$
\begin{aligned}
& \left(\bar{x}_{1}, \bar{y}_{1}\right)=\left(\frac{1-\sqrt{5}}{2}, \frac{1-\sqrt{5}}{2}\right), \\
& \left(\bar{x}_{2}, \bar{y}_{2}\right)=\left(\frac{1+\sqrt{5}}{2}, \frac{1+\sqrt{5}}{2}\right) .
\end{aligned}
$$

Owing to $\frac{1+\sqrt{5}}{2} \approx 1.618$, the elements of second equilibrium point is the Golden Ratio.

Proof. We can easily seen for the equilibrium points of system (1.1):

$$
\begin{aligned}
& \bar{x}=\bar{x} \cdot \bar{y}-1, \\
& \bar{y}=\bar{y} \cdot \bar{x}-1 .
\end{aligned}
$$


From this system, we obtain

$$
\bar{x}=\bar{x} \cdot \bar{y}-1=\bar{y}
$$

Thus

$$
\begin{aligned}
& \bar{x}=\bar{x} \cdot \bar{x}-1 \\
& \bar{x}=\bar{x}^{2}-1 \\
& \bar{x}=\frac{1 \pm \sqrt{5}}{2}
\end{aligned}
$$

So we finished the proof as desired.

\section{Periodic Solutions of System (1.1)}

In this section, we investigate the periodic solutions of system (1.1) which are two periodic, three periodic and eventually three periodic.

Theorem 3. System (1.1) has periodic solutions with period two, if and only if $x_{-1}=\bar{x}_{1}, x_{0}=\bar{x}_{2}, y_{-1}=\bar{y}_{2}, y_{0}=\bar{y}_{1}$ or $x_{-1}=\bar{x}_{2}, x_{0}=\bar{x}_{1}, y_{-1}=\bar{y}_{1}, y_{0}=\bar{y}_{2}$, they will be taken following forms:

$$
\left\{\left(\bar{x}_{1}, \bar{y}_{2}\right),\left(\bar{x}_{2}, \bar{y}_{1}\right),\left(\bar{x}_{1}, \bar{y}_{2}\right), \cdots\right\}
$$

or

$$
\left\{\left(\bar{x}_{2}, \bar{y}_{1}\right),\left(\bar{x}_{1}, \bar{y}_{2}\right),\left(\bar{x}_{2}, \bar{y}_{1}\right), \cdots\right\}
$$

Proof. If the initial values are as given, then by some calculations it is clear to see that these solutions are of period two. Let $\left\{\left(x_{n}, y_{n}\right)\right\}_{n=-1}^{\infty}$ be a periodic solution of system (1.1) with period two. Therefore, $x_{2 n}=a, x_{2 n-1}=b, y_{2 n}=c$ and $y_{2 n-1}=d$ for every $n \in \mathbb{N}_{0}$ and there exist some $a, b, c, d$ such that $a \neq b$ and $c \neq d$. Thus we have from system (1.1):

$$
\begin{aligned}
& x_{1}=x_{-1} y_{0}-1=b \cdot c-1=b \\
& y_{1}=y_{-1} x_{0}-1=d \cdot a-1=d \\
& x_{2}=x_{0} y_{1}-1=a \cdot d-1=a \\
& y_{2}=y_{0} x_{1}-1=c \cdot b-1=c .
\end{aligned}
$$

From (3.1)-(3.4), we get that:

$$
\begin{aligned}
a & =\bar{x}_{1}, b=\bar{x}_{2}, c=\bar{y}_{2}, d=\bar{y}_{1}, \\
a & =\bar{x}_{2}, b=\bar{x}_{1}, c=\bar{y}_{1}, d=\bar{y}_{2}, \\
a & =b=\bar{x}_{1}, c=d=\bar{y}_{1}, \\
a & =b=\bar{x}_{2}, c=d=\bar{y}_{2} .
\end{aligned}
$$

As a result, (3.5) and (3.6) are periodic solutions of system (1.1) with period two but (3.7) and (3.8) are not periodic solutions of system (1.1) with period two. Because they are trivial solutions of system (1.1). The proof completed.

Remark 1. From (3.5) and (3.6), system (1.1) has a two periodic cycle as:

$$
\left\{\cdots,\left(\bar{x}_{1}, \bar{y}_{2}\right),\left(\bar{x}_{2}, \bar{y}_{1}\right),\left(\bar{x}_{1}, \bar{y}_{2}\right), \cdots\right\}
$$


Proof. Let $x_{-1}=\bar{x}_{1}, x_{0}=\bar{x}_{2}, y_{-1}=\bar{y}_{2}, y_{0}=\bar{y}_{1}$. Hence, we have from system (1.1):

$$
\begin{aligned}
& x_{1}=x_{-1} y_{0}-1=\bar{x}_{1} \cdot \bar{y}_{1}-1=\bar{x}_{1} \\
& y_{1}=y_{-1} x_{0}-1=\bar{y}_{2} \cdot \bar{x}_{2}-1=\bar{y}_{2} \\
& x_{2}=x_{0} y_{1}-1=\bar{x}_{2} \cdot \bar{y}_{2}-1=\bar{x}_{2} \\
& y_{2}=y_{0} x_{1}-1=\bar{y}_{1} \cdot \bar{x}_{1}-1=\bar{y}_{1} .
\end{aligned}
$$

Consequently, two periodic cycle of system (1.1) has been completed as desired.

Theorem 4. System (1.1) has periodic solutions with period three, if and only if the initial values are

$$
\begin{aligned}
& x_{-1}=0, x_{0}=-1, y_{-1}=0, y_{0}=-1, \\
& x_{-1}=-1, x_{0}=-1, y_{-1}=-1, y_{0}=-1, \\
& x_{-1}=-1, x_{0}=0, y_{-1}=-1, y_{0}=0 .
\end{aligned}
$$

Proof. If the initial values are as given, then by some calculations it is clear to see that these solutions are of period three. Let $\left\{\left(x_{n}, y_{n}\right)\right\}_{n=-1}^{\infty}$ be a periodic solution of system (1.1) with period three. Thus, we take $x_{-1}=a, x_{0}=b, y_{-1}=c, y_{0}=d$. Therefore, we have from system (1.1),

$$
\begin{aligned}
& x_{1}=x_{-1} y_{0}-1=a \cdot d-1, \\
& y_{1}=y_{-1} x_{0}-1=c \cdot b-1, \\
& x_{2}=x_{0} y_{1}-1=b \cdot(c \cdot b-1)-1=a, \\
& y_{2}=y_{0} x_{1}-1=d \cdot(a \cdot d-1)-1=c, \\
& x_{3}=x_{1} y_{2}-1=(a \cdot d-1) \cdot c-1=b, \\
& y_{3}=y_{1} x_{2}-1=(c \cdot b-1) \cdot a-1=d .
\end{aligned}
$$

From (3.12)-(3.15) we obtain that

$$
\begin{aligned}
c b^{2}-b-1 & =a \\
a d^{2}-d-1 & =c \\
a c d-c-1 & =b \\
a b c-a-1 & =d .
\end{aligned}
$$

From (3.16)-(3.19), we get

$$
\begin{aligned}
& a=0, b=-1, c=0, d=-1, \\
& a=-1, b=-1, c=-1, d=-1, \\
& a=-1, b=0, c=-1, d=0 .
\end{aligned}
$$

So, the proof completed.

Remark 2. From (3.9)-(3.11), system (1.1) has a three periodic cycle as:

$$
\{\cdots,(-1,-1),(0,0),(-1,-1),(-1,-1), \cdots\} \text {. }
$$


Proof. Suppose that the initial values are $x_{-1}=0, x_{0}=-1, y_{-1}=0, y_{0}=-1$. Thus, we get from system (1.1):

$$
\begin{aligned}
& x_{1}=x_{-1} y_{0}-1=0 \cdot(-1)-1=-1, \\
& y_{1}=y_{-1} x_{0}-1=0 \cdot(-1)-1=-1, \\
& x_{2}=x_{0} y_{1}-1=(-1) \cdot(-1)-1=0, \\
& y_{2}=y_{0} x_{1}-1=(-1) \cdot(-1)-1=0, \\
& x_{3}=x_{1} y_{2}-1=(-1) \cdot 0-1=-1, \\
& y_{3}=y_{1} x_{2}-1=(-1) \cdot 0-1=-1, \\
& x_{4}=x_{2} y_{3}-1=0 \cdot(-1)-1=-1, \\
& y_{4}=y_{2} x_{3}-1=0 \cdot(-1)-1=-1 .
\end{aligned}
$$

From these we obtain three periodic cycle of system (1.1) as:

$$
\{(-1,-1),(0,0),(-1,-1),(-1,-1), \cdots\} \text {. }
$$

Hence, the proof completed.

Theorem 5. There is eventually periodic solution of system (1.1) with period three as:

$$
\left\{\left(x_{-1}, y_{-1}\right),\left(x_{0}, y_{0}\right), \cdots,\left(x_{n-1}, y_{n-1}\right),\left(x_{n}, y_{n}\right),(0,0),(-1,-1),(-1,-1), \cdots\right\}
$$

where $x_{n-1} y_{n}=1$ and $y_{n-1} x_{n}=1$ for $n \in \mathbb{N}_{0}$.

Proof. Let $\left\{\left(x_{n}, y_{n}\right)\right\}_{n=-1}^{\infty}$ be a eventually periodic solution of system (1.1) with period three. By means of Theorem 4, we take

$$
\begin{aligned}
& \left(x_{n+1}, y_{n+1}\right)=(0,0), \\
& \left(x_{n+2}, y_{n+2}\right)=(-1,-1) .
\end{aligned}
$$

From these, we obtain that

$$
\begin{aligned}
x_{n+1} & =x_{n-1} y_{n}-1=0, \\
y_{n+1} & =y_{n-1} x_{n}-1=0, \\
x_{n+2} & =x_{n} y_{n+1}-1=-1, \\
y_{n+2} & =y_{n} x_{n+1}-1=-1 .
\end{aligned}
$$

Therefore, we have $x_{n-1} y_{n}=1$ and $y_{n-1} x_{n}=1$. Moreover, due to $x_{n+1}=0$ and $y_{n+1}=0$, the following equailities satisfy under all conditions: $x_{n+2}=-1$ and $y_{n+2}=-1$. Thus, the proof finished.

\section{Unbounded Solutions of System (1.1)}

In this section, we study the unbounded solutions of system (1.1).

Lemma 1. Let $\left\{\left(x_{n}, y_{n}\right)\right\}_{n=-1}^{\infty}$ be a solution of system (1.1). Let

$$
x_{-1}<-1, x_{0}<-1, y_{-1}<-1 \text { and } y_{0}<-1 \text {. }
$$

Then

$$
\begin{gathered}
x_{1}>0, \quad y_{1}>0, \\
x_{2}<-1, \quad y_{2}<-1, \\
x_{3}<-1, \quad y_{3}<-1 .
\end{gathered}
$$


Proof. Let $\left\{\left(x_{n}, y_{n}\right)\right\}_{n=-1}^{\infty}$ be a solution of system (1.1). Suppose that $x_{-1}<$ $-1, x_{0}<-1, y_{-1}<-1$ and $y_{0}<-1$. From these and system (1.1) we have,

$$
\begin{aligned}
& x_{1}=x_{-1} y_{0}-1>0, \\
& y_{1}=y_{-1} x_{0}-1>0, \\
& x_{2}=x_{0} y_{1}-1<-1, \\
& y_{2}=y_{0} x_{1}-1<-1, \\
& x_{3}=x_{1} y_{2}-1<-1, \\
& y_{3}=y_{1} x_{2}-1<-1 .
\end{aligned}
$$

The next theorem has an important role for Theorem 7.

Theorem 6. Let $\left\{\left(x_{n}, y_{n}\right)\right\}_{n=-1}^{\infty}$ be a solution of system (1.1). Then,

$$
\begin{aligned}
& x_{n+3}-y_{n}=\left(x_{n+1}+1\right)\left(y_{n+2}-y_{n}\right), \\
& y_{n+3}-x_{n}=\left(y_{n+1}+1\right)\left(x_{n+2}-x_{n}\right) .
\end{aligned}
$$

Proof. Let $\left\{\left(x_{n}, y_{n}\right)\right\}_{n=-1}^{\infty}$ be a solution of system (1.1). We have from system $(1.1)$

$$
\begin{aligned}
x_{n+3}-y_{n} & =\left(x_{n+1} y_{n+2}-1\right)-y_{n} \\
& =x_{n+1}\left(y_{n} x_{n+1}-1\right)-1-y_{n} \\
& =x_{n+1}^{2} y_{n}-x_{n+1}-1-y_{n} \\
& =y_{n}\left(x_{n+1}^{2}-1\right)-\left(x_{n+1}+1\right) \\
& =\left(x_{n+1}+1\right)\left(y_{n} x_{n+1}-y_{n}-1\right) \\
& =\left(x_{n+1}+1\right)\left(y_{n+2}-y_{n}\right) .
\end{aligned}
$$

Likewise, (4.2) can be proved. Therefore, the proof completed.

Theorem 7. Let $\left\{\left(x_{n}, y_{n}\right)\right\}_{n=-1}^{\infty}$ be a solution of system (1.1). Let

$$
x_{-1}<-1, x_{0}<-1, y_{-1}<-1 \text { and } y_{0}<-1 \text {. }
$$

Then the following two statements hold, for $n=0,1,2, \cdots$, :

(i):

$$
\begin{array}{r}
0<x_{1}<y_{4}<x_{7}<\cdots<x_{6 n+1}<y_{6 n+4}<\cdots, \\
0<y_{1}<x_{4}<y_{7}<\cdots<y_{6 n+1}<x_{6 n+4}<\cdots, \\
-1>x_{2}>y_{5}>x_{8}>\cdots>x_{6 n+2}>y_{6 n+5}>\cdots, \\
-1>y_{2}>x_{5}>y_{8}>\cdots>y_{6 n+2}>x_{6 n+5}>\cdots, \\
-1>x_{3}>y_{6}>x_{9}>\cdots>x_{6 n+3}>y_{6 n+6}>\cdots, \\
-1>y_{3}>x_{6}>y_{9}>\cdots>y_{6 n+3}>x_{6 n+6}>\cdots
\end{array}
$$


(ii):

$$
\begin{array}{cc}
\lim _{n \rightarrow \infty} x_{6 n+1}=\infty, & \lim _{n \rightarrow \infty} y_{6 n+1}=\infty, \\
\lim _{n \rightarrow \infty} x_{6 n+2}=-\infty, & \lim _{n \rightarrow \infty} y_{6 n+2}=-\infty, \\
\lim _{n \rightarrow \infty} x_{6 n+3}=-\infty, & \lim _{n \rightarrow \infty} y_{6 n+3}=-\infty \\
\lim _{n \rightarrow \infty} x_{6 n+4}=\infty, & \lim _{n \rightarrow \infty} y_{6 n+4}=\infty \\
\lim _{n \rightarrow \infty} x_{6 n+5}=-\infty, & \lim _{n \rightarrow \infty} y_{6 n+5}=-\infty \\
\lim _{n \rightarrow \infty} x_{6 n}=-\infty, & \lim _{n \rightarrow \infty} y_{6 n}=-\infty
\end{array}
$$

Proof. Let $\left\{\left(x_{n}, y_{n}\right)\right\}_{n=-1}^{\infty}$ be a solution of system (1.1). Let $x_{-1}<-1, x_{0}<$ $-1, y_{-1}<-1$ and $y_{0}<-1$.

(i) From Lemma 1, we know that $x_{1}, y_{1}>0, x_{2}, y_{2}<-1$ and $x_{3}, y_{3}<-1$.

From Theorem 6 for $n=1$, we obtain,

$$
x_{4}-y_{1}=\left(x_{2}+1\right)\left(y_{3}-y_{1}\right) .
$$

From $x_{2}, y_{3}<-1$ and $y_{1}>0$, we get that

$$
x_{4}-y_{1}>0 .
$$

So, $x_{4}>y_{1}>0$ and similarly $y_{4}>x_{1}>0$.

From Theorem 6 for $n=2$, we have that,

$$
x_{5}-y_{2}=\left(x_{3}+1\right)\left(y_{4}-y_{2}\right) .
$$

From $y_{2}, x_{3}<-1$ and $y_{4}>0$, we obtain,

$$
x_{5}-y_{2}<0 .
$$

Thus, $x_{5}<y_{2}<-1$ and further $y_{5}<x_{2}<-1$.

From Theorem 6 for $n=3$, we obtain,

$$
x_{6}-y_{3}=\left(x_{4}+1\right)\left(y_{5}-y_{3}\right) \text {. }
$$

From system (1.1), we get,

$$
\begin{aligned}
x_{6}-y_{3} & =\left(x_{4}+1\right)\left(y_{5}-y_{3}\right) \\
& =\left(x_{4}+1\right)\left(y_{3} x_{4}-1-y_{3}\right) \\
& =\left(x_{4}+1\right)\left(y_{3}\left(x_{2} y_{3}-1\right)-1-y_{3}\right) \\
& =\left(x_{4}+1\right)\left(x_{2} y_{3}^{2}-2 y_{3}-1\right)
\end{aligned}
$$

From $x_{2}<-1$,

$$
\begin{aligned}
x_{6}-y_{3} & =\left(x_{4}+1\right)\left(x_{2} y_{3}^{2}-2 y_{3}-1\right) \\
& <\left(x_{4}+1\right)\left(-y_{3}^{2}-2 y_{3}-1\right) \\
& =\left(x_{4}+1\right)\left(-\left(y_{3}+1\right)^{2}\right)
\end{aligned}
$$

From $x_{4}>0$ and $y_{3}<-1$ we have

$$
x_{6}-y_{3}<0 .
$$

Hence, $x_{6}<y_{3}<-1$ and likewise $y_{6}<x_{3}<-1$.

From Theorem 6 for $n=4$, we have,

$$
x_{7}-y_{4}=\left(x_{5}+1\right)\left(y_{6}-y_{4}\right) .
$$

From $y_{6}, x_{5}<-1$ and $y_{4}>0$, we obtain,

$$
x_{7}-y_{4}>0 \text {. }
$$


So, $x_{7}>y_{4}>x_{1}>0$ and similarly $y_{7}>x_{4}>y_{1}>0$.

In addition, we have by induction:

$$
\begin{aligned}
0 & <x_{1}<y_{4}<x_{7}<\cdots, \\
0 & <y_{1}<x_{4}<y_{7}<\cdots, \\
-1 & >x_{2}>y_{5}>x_{8}>\cdots, \\
-1 & >y_{2}>x_{5}>y_{8}>\cdots, \\
-1 & >x_{3}>y_{6}>x_{9}>\cdots, \\
-1 & >y_{3}>x_{6}>y_{9}>\cdots .
\end{aligned}
$$

Thus, proof of (i) finished.

(ii)

$$
\begin{aligned}
x_{6 n+1} & =x_{6 n-1} y_{6 n}-1 \\
& =\left(x_{6 n-3} y_{6 n-2}-1\right)\left(y_{6 n-2} x_{6 n-1}-1\right)-1 \\
& =x_{6 n-3} y_{6 n-2}^{2} x_{6 n-1}-y_{6 n-2} x_{6 n-1}-x_{6 n-3} y_{6 n-2}+1-1
\end{aligned}
$$

From $x_{6 n-3} y_{6 n-2}^{2} x_{6 n-1}>0$ and $y_{6 n-2} x_{6 n-1}<0$, we obtain

$$
x_{6 n+1}>-x_{6 n-3} y_{6 n-2}
$$

From $x_{6 n-3}<-1$, we have

$$
\begin{aligned}
x_{6 n+1} & >y_{6 n-2}=y_{6 n-4} x_{6 n-3}-1 \\
& =y_{6 n-4}\left(x_{6 n-5} y_{6 n-4}-1\right)-1 \\
& =x_{6 n-5} y_{6 n-4}^{2}-y_{6 n-4}-1
\end{aligned}
$$

From $y_{6 n-4}<-1$, we get

$$
x_{6 n+1}>x_{6 n-5}
$$

So,

$$
\lim _{n \rightarrow \infty} x_{6 n+1}=\infty .
$$

The proof of the other cases is similar to this, therefore we leave it to readers.

Theorem 8. Let $\left\{\left(x_{n}, y_{n}\right)\right\}_{n=-1}^{\infty}$ be a solution of system (1.1). Let $x_{-1}, x_{0}>\bar{x}_{2}$ and $y_{-1}, y_{0}>\bar{y}_{2}$. Then the following two statements hold for $n=0,1,2, \cdots$,

(i):

$$
\begin{aligned}
& \bar{x}_{2}<x_{0}<x_{2}<x_{4}<\cdots<x_{2 n}<\cdots, \\
& \bar{x}_{2}<x_{1}<x_{3}<x_{5}<\cdots<x_{2 n-1}<\cdots, \\
& \bar{y}_{2}<y_{0}<y_{2}<y_{4}<\cdots<y_{2 n}<\cdots, \\
& \bar{y}_{2}<y_{1}<y_{3}<y_{5}<\cdots<y_{2 n-1}<\cdots .
\end{aligned}
$$

(ii): The subsequences $\left\{x_{2 n}\right\}_{n=0}^{\infty},\left\{x_{2 n-1}\right\}_{n=0}^{\infty},\left\{y_{2 n}\right\}_{n=0}^{\infty}$ and $\left\{y_{2 n-1}\right\}_{n=0}^{\infty}$ tend to $+\infty$.

Proof. Let $\left\{\left(x_{n}, y_{n}\right)\right\}_{n=-1}^{\infty}$ be a solution of system (1.1). Then we have,

$$
\begin{aligned}
& x_{0}>\frac{1+\sqrt{5}}{2}, \\
& y_{0}>\frac{1+\sqrt{5}}{2} .
\end{aligned}
$$


Therefore,

$$
\frac{1}{x_{0}}<\frac{2}{1+\sqrt{5}}=\frac{\sqrt{5}-1}{2}
$$

Hence we get,

$$
\begin{aligned}
1+\frac{1}{x_{0}} & <1+\frac{\sqrt{5}-1}{2}=\frac{1+\sqrt{5}}{2}<y_{-1}, \\
1+\frac{1}{x_{0}} & <y_{-1} .
\end{aligned}
$$

Thus,

$$
\begin{aligned}
x_{0}+1 & <y_{-1} x_{0}, \\
x_{0} & <y_{-1} x_{0}-1 .
\end{aligned}
$$

So, $\bar{x}_{2}<x_{0}<y_{1}$ and similarly $\bar{y}_{2}<y_{0}<x_{1}$.

From these,

$$
\begin{aligned}
\frac{1}{x_{1}} & <\frac{2}{1+\sqrt{5}}=\frac{\sqrt{5}-1}{2} \\
1+\frac{1}{x_{1}} & <1+\frac{\sqrt{5}-1}{2}=\frac{1+\sqrt{5}}{2}<y_{0} .
\end{aligned}
$$

Therefore we have

$$
\begin{aligned}
1+\frac{1}{x_{1}} & <y_{0}, \\
x_{1}+1 & <y_{0} x_{1}, \\
x_{1} & <y_{0} x_{1}-1 .
\end{aligned}
$$

Hence, $x_{1}<y_{2}$ and similarly $y_{1}<x_{2}$.

So, we have by induction

$$
\begin{aligned}
& \bar{x}_{2}=\bar{y}_{2}<y_{0}<x_{1}<y_{2}<x_{3}<\cdots, \\
& \bar{x}_{2}=\bar{y}_{2}<x_{0}<y_{1}<x_{2}<y_{3}<\cdots .
\end{aligned}
$$

Thus proof of (i) finished. Now we begin to proof of (ii).

We have from (i), the subsequences $\left\{x_{2 n}\right\}_{n=0}^{\infty},\left\{x_{2 n-1}\right\}_{n=0}^{\infty},\left\{y_{2 n}\right\}_{n=0}^{\infty}$ and $\left\{y_{2 n-1}\right\}_{n=0}^{\infty}$ are strictly increasing. For the sake of contradiction, these susequences have finite limits. Since the subsequences are increasing, they must be bounded from above. But the system (1.1) has two equilibrium points such that they are less than initial values. That is a contradiction as desired.

\section{Invariant of System (1.1)}

Theorem 9. Let all initial values of system (1.1) in $(-1,0)$. Then the solution of system (1.1) is such that $x_{n} \in(-1,0)$ and $y_{n} \in(-1,0)$ for all $n \geq-1$.

Proof. Let $x_{-1}, x_{0}, y_{-1}, y_{0} \in(-1,0)$. We can clearly seen from system (1.1):

$$
\begin{aligned}
& x_{1}=x_{-1} y_{0}-1 \in(-1,0), \\
& y_{1}=y_{-1} x_{0}-1 \in(-1,0) .
\end{aligned}
$$

Thus we have following:

$$
\begin{aligned}
& x_{2}=x_{0} y_{1}-1 \in(-1,0), \\
& y_{2}=y_{0} x_{1}-1 \in(-1,0) .
\end{aligned}
$$


So, by using (5.1)-(5.4) and induction, we get $x_{n} \in(-1,0)$ and $y_{n} \in(-1,0)$ for all $n \geq-1$.

\section{Stability Analysis of System (1.1)}

In this section, we investigate the stability of system (1.1). Further, we find out negative equilibrium point of system (1.1) is locally asymptotically stable and if initial values are in $(-1,0)$, then solutions of system (1.1) converge to negative equilibrium point. But we discover the positive equilibrium point of system (1.1) is unstable.

Theorem 10. Negative equilibrium point $\left(\bar{x}_{1}, \bar{y}_{1}\right)$ of system (1.1) is locally asymptotically stable.

Proof. System (1.1) is equivalent to following system of difference equations:

$$
t_{n+1}=1-t_{n-1} w_{n}, w_{n+1}=1-w_{n-1} t_{n}, n=0,1,2, \ldots,
$$

with change to variables $x_{n}=-t_{n}$ and $y_{n}=-w_{n}$. From this, negative equilibrium point $\left(\bar{x}_{1}, \bar{y}_{1}\right)$ of system turn to positive equilibrium point $(\bar{t}, \bar{w})$ of system $(6.1)$. We can clearly seen that

$$
(\bar{t}, \bar{w})=\left(\frac{\sqrt{5}-1}{2}, \frac{\sqrt{5}-1}{2}\right) .
$$

Now we study linearized form of system (6.1). For this, we consider the transformation:

$$
\left(t_{n}, t_{n-1}, w_{n}, w_{n-1}\right) \rightarrow\left(h, h_{1}, k, k_{1}\right)
$$

where

$$
\begin{aligned}
h & =1-t_{n-1} w_{n}, \\
h_{1} & =t_{n}, \\
k & =1-w_{n-1} t_{n}, \\
k_{1} & =w_{n} .
\end{aligned}
$$

Therefore we have the Jacobian matrix about equilibrium point $(\bar{t}, \bar{w})$ :

$$
B(\bar{t}, \bar{w})=\left(\begin{array}{cccc}
0 & -\bar{w} & -\bar{t} & 0 \\
1 & 0 & 0 & 0 \\
-\bar{w} & 0 & 0 & -\bar{t} \\
0 & 0 & 1 & 0
\end{array}\right) .
$$

Thus, the linearized system about the equilibrium point $(\bar{t}, \bar{w})=\left(\frac{\sqrt{5}-1}{2}, \frac{\sqrt{5}-1}{2}\right)$ is $X_{N+1}=B(\bar{t}, \bar{w}) X_{n}$, where $X_{n}=\left(\left(t_{n}, t_{n-1}, w_{n}, w_{n-1}\right)\right)^{T}$ and

$$
B(\bar{t}, \bar{w})=\left(\begin{array}{cccc}
0 & \frac{1-\sqrt{5}}{2} & \frac{1-\sqrt{5}}{2} & 0 \\
1 & 0 & 0 & 0 \\
\frac{1-\sqrt{5}}{2} & 0 & 0 & \frac{1-\sqrt{5}}{2} \\
0 & 0 & 1 & 0
\end{array}\right) .
$$

So, the characteristic equation of $B(\bar{t}, \bar{w})$ is

$$
\lambda^{4}-\left(\frac{5-3 \sqrt{5}}{2}\right) \lambda^{2}+\frac{3-\sqrt{5}}{2}=0 \text {. }
$$


Hence, we have four roots of Eq.(6.2):

$$
\begin{gathered}
\lambda_{1}=\frac{1}{2} \sqrt{5-3 \sqrt{5}-2 \sqrt{\frac{23}{2}-\frac{11}{2} \sqrt{5}}}=0.30902-0.72287 i \\
\lambda_{2}=-\frac{1}{2} \sqrt{5-3 \sqrt{5}-2 \sqrt{\frac{23}{2}-\frac{11}{2} \sqrt{5}}}=-0.30902+0.72287 i \\
\lambda_{3}=\frac{1}{2} \sqrt{2 \sqrt{\frac{23}{2}-\frac{11}{2} \sqrt{5}}-3 \sqrt{5}+5}=0.30902+0.72287 i \\
\lambda_{4}=-\frac{1}{2} \sqrt{2 \sqrt{\frac{23}{2}-\frac{11}{2} \sqrt{5}}-3 \sqrt{5}+5}=-0.30902-0.72287 i
\end{gathered}
$$

Due to

$$
\left|\lambda_{1}\right|=\left|\lambda_{2}\right|=\left|\lambda_{3}\right|=\left|\lambda_{4}\right|=0.78615<1,
$$

and from linearized stability theorem, all roots of the characteristic equation lie inside the unit disk. So, the positive equilibrium of system (6.1) is locally asymptotically stable. Therefore, the negative equilibrium of system (1.1) is locally asymptotically stable as desired.

Theorem 11. Let $x_{-1}, x_{0}, y_{-1}, y_{0} \in(-1,0)$. Then every solutions of system (1.1) converge to negative equilibrium point $\left(\bar{x}_{1}, \bar{y}_{1}\right)$ of related system.

Proof. Let $x_{-1}, x_{0}, y_{-1}, y_{0} \in(-1,0)$. Then, from Theorem 9, every solutions of system (1.1) is bounded. Additionally, we consider system (6.1) from Theorem 10's proof. Hence, we have that if $x_{-1}, x_{0}, y_{-1}, y_{0} \in(-1,0)$ then $t_{-1}, t_{0}, w_{-1}, w_{0} \in(0,1)$. Therefore, $t_{n} \in(0,1)$ and $w_{n} \in(0,1)$ for $n \geq-1$. Thus, we take

$$
\begin{aligned}
L_{1} & =\lim _{n \rightarrow \infty} \sup t_{n}, & L_{2} & =\lim _{n \rightarrow \infty} \sup w_{n}, \\
l_{1} & =\lim _{n \rightarrow \infty} \inf t_{n}, & l_{2} & =\lim _{n \rightarrow \infty} \inf w_{n} .
\end{aligned}
$$

Thus, we obtain from system (1.1) and (6.3):

$$
\begin{aligned}
L_{1} & \leq 1-L_{1} \cdot l_{2}, \\
l_{1} & \geq 1-l_{1} \cdot L_{2}, \\
L_{2} & \leq 1-L_{2} \cdot l_{1}, \\
l_{2} & \geq 1-l_{2} \cdot L_{1} .
\end{aligned}
$$

Hence we have from (6.4) and (6.7)

$$
1-l_{2} \leq L_{1} \cdot l_{2} \leq 1-L_{1}
$$

So

$$
L_{1} \leq l_{2} .
$$

Similarly, we have from (6.5) and (6.6)

$$
L_{2} \leq l_{1}
$$

In addition, we know that,

$$
l_{1} \leq L_{1} \text { and } l_{2} \leq L_{2} .
$$

Therefore, we get from (6.8), (6.9) and (6.10)

$$
l_{1} \leq L_{1} \leq l_{2} \leq L_{2} \leq l_{1} .
$$


So, we obtain that

$$
l_{1}=L_{1}=l_{2}=L_{2} .
$$

Thus, if the initial values lie in $(0,1)$ then the every solution $\left\{\left(t_{n}, w_{n}\right)\right\}$ of system (6.1) tends to positive equilibrium point $(\bar{t}, \bar{w})$ of system (6.1). Due to the fact that positive equilibrium point $(\bar{t}, \bar{w})$ of system $(6.1)$ is negative equilibrium point $\left(\bar{x}_{1}, \bar{y}_{1}\right)$ of system (1.1), every solution of system (1.1) tends to negative equilibrium point $\left(\bar{x}_{1}, \bar{y}_{1}\right)$ which the initial values in $(-1,0)$. So, the proof completed as desired.

Theorem 12. Equilibrium point $\left(\bar{x}_{2}, \bar{y}_{2}\right)$ of system (1.1) is locally unstable.

Proof. Firstly we study linearized form of system (1.1). For this, we consider the transformation:

$$
\left(x_{n}, x_{n-1}, y_{n}, y_{n-1}\right) \rightarrow\left(f, f_{1}, g, g_{1}\right)
$$

where

$$
\begin{aligned}
f & =x_{n-1} y_{n}-1, \\
f_{1} & =x_{n}, \\
g & =y_{n-1} x_{n}-1, \\
g_{1} & =y_{n} .
\end{aligned}
$$

Therefore we have the Jacobian matrix about equilibrium point $(\bar{x}, \bar{y})$ :

$$
B(\bar{x}, \bar{y})=\left(\begin{array}{cccc}
0 & \bar{y} & \bar{x} & 0 \\
1 & 0 & 0 & 0 \\
\bar{y} & 0 & 0 & \bar{x} \\
0 & 0 & 1 & 0
\end{array}\right)
$$

Thus, the linearized system about the equilibrium point $(\bar{x}, \bar{y})=\left(\frac{1+\sqrt{5}}{2}, \frac{1+\sqrt{5}}{2}\right)$ is $X_{N+1}=B(\bar{x}, \bar{y}) X_{n}$ where $X_{n}=\left(\left(x_{n}, x_{n-1}, y_{n}, y_{n-1}\right)\right)^{T}$ and

$$
B(\bar{x}, \bar{y})=\left(\begin{array}{cccc}
0 & \frac{1+\sqrt{5}}{2} & \frac{1+\sqrt{5}}{2} & 0 \\
1 & 0 & 0 & 0 \\
\frac{1+\sqrt{5}}{2} & 0 & 0 & \frac{1+\sqrt{5}}{2} \\
0 & 0 & 1 & 0
\end{array}\right) .
$$

So, the characteristic equation of $B(\bar{x}, \bar{y})$ is

$$
\lambda^{4}-\left(\frac{5+3 \sqrt{5}}{2}\right) \lambda^{2}+\frac{3+\sqrt{5}}{2}=0 .
$$

Hence, we have four roots of Eq.(6.11):

$$
\begin{aligned}
& \lambda_{1}=\frac{1}{2} \sqrt{3 \sqrt{5}-2 \sqrt{\frac{11}{2} \sqrt{5}+\frac{23}{2}}+5}=0.69848 \\
& \lambda_{2}=-\frac{1}{2} \sqrt{3 \sqrt{5}-2 \sqrt{\frac{11}{2} \sqrt{5}+\frac{23}{2}}+5}=-0.69848 \\
& \lambda_{3}=\frac{1}{2} \sqrt{2 \sqrt{\frac{11}{2} \sqrt{5}+\frac{23}{2}}+3 \sqrt{5}+5}=2.3165, \\
& \lambda_{4}=\frac{1}{2} \sqrt{2 \sqrt{\frac{11}{2} \sqrt{5}+\frac{23}{2}}+3 \sqrt{5}+5}=-2.3165 .
\end{aligned}
$$


Because of

$$
\left|\lambda_{1,2}\right|<1<\left|\lambda_{3,4}\right|
$$

and from linearized stability theorem, two roots of the characteristic equation lie inside the unit disk but the other roots lie outside the unit disk. So, the positive equilibrium of system (1.1) is locally unstable.

\section{Numerical Examples}

Now, we present some numerical examples for verify our theoretical results of previous sections.

Example 1. Consider the system (1.1) with initial values $x_{-1}=\bar{x}_{1}, x_{0}=\bar{x}_{2}, y_{-1}=$ $\bar{y}_{2}, y_{0}=\bar{y}_{1}$. Then, the system (1.1) has periodic solution with prime period two. The Figure 1 verifies our theoretical results (See Theorem 3).

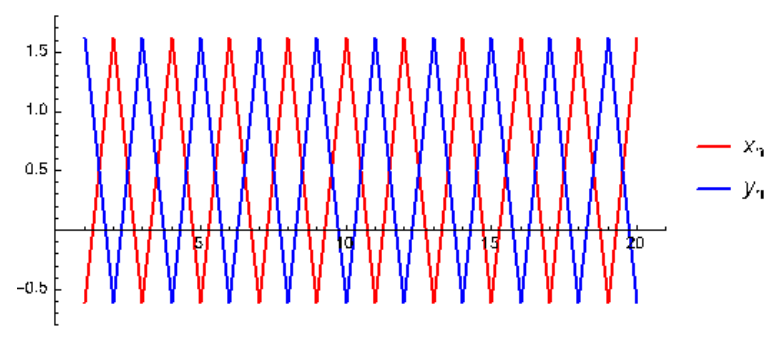

Figure 1. Plot of system (1.1) for initial values $x_{-1}=\bar{x}_{1}, x_{0}=$ $\bar{x}_{2}, y_{-1}=\bar{y}_{2}, y_{0}=\bar{y}_{1}$.

Example 2. Consider the system (1.1) with initial values $x_{-1}=0, x_{0}=-1, y_{-1}=$ $0, y_{0}=-1$. Then, the system (1.1) has periodic solution with period three. The figure 2 and figure 3 verify our theoretical results (See Theorem 4).

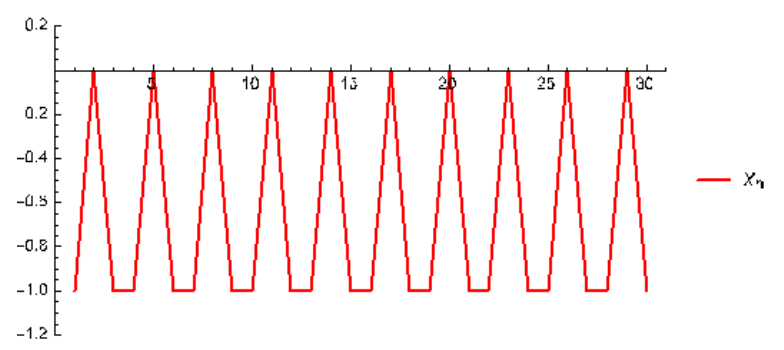

Figure 2. Plot of $x_{n}$ for initial values $x_{-1}=0, x_{0}=-1, y_{-1}=0$, $y_{0}=-1$.

Example 3. Consider the system (1.1) with initial values $x_{-1}=\frac{3248}{5}, x_{0}=$ $\frac{208}{1539}, y_{-1}=\frac{287793}{3328}, y_{0}=\frac{5}{112}$. Then, the system (1.1) has eventually periodic solution with period three. The figure 4 and figure 5 verify our theoretical results (See Theorem 5).

Example 4. Consider the system (1.1) with initial values $x_{-1}=-0.1, x_{0}=$ $-0.8, y_{-1}=-0.9, y_{0}=-0.2$. Then, solution of system (1.1) converges to negative equilibrium point $\left(\bar{x}_{1}, \bar{y}_{1}\right)$. The Figure 6 verifies our theoretical results (See Theorem 11). 


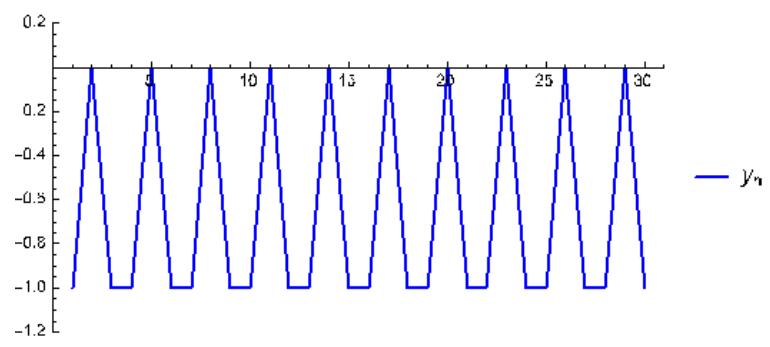

Figure 3. Plot of $y_{n}$ for initial values $x_{-1}=0, x_{0}=-1, y_{-1}=0$, $y_{0}=-1$.

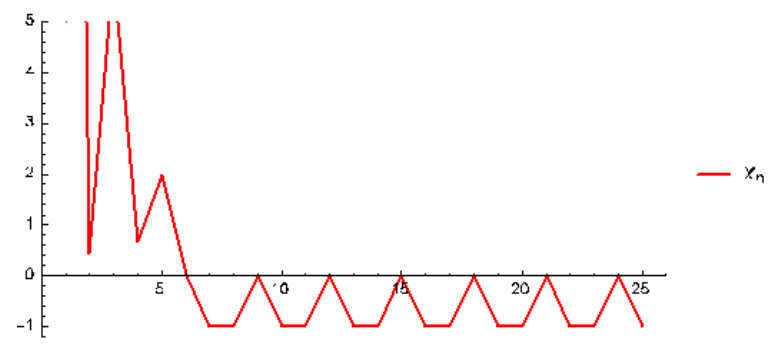

Figure 4. Plot of $x_{n}$ for initial values initial values $x_{-1}=\frac{3248}{5}$, $x_{0}=\frac{208}{1539}, y_{-1}=\frac{287793}{3328}, y_{0}=\frac{5}{112}$.

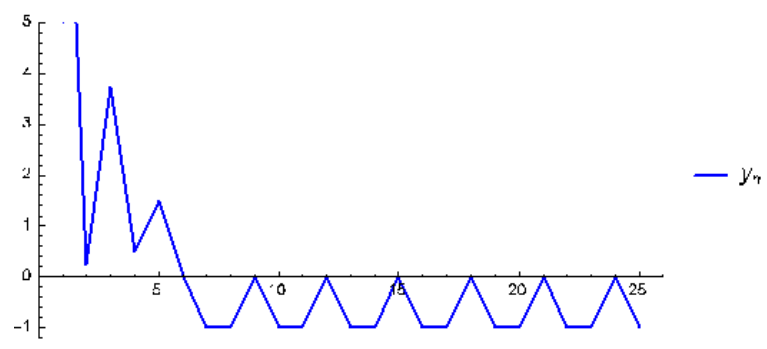

Figure 5. Plot of $y_{n}$ for initial values initial values $x_{-1}=\frac{3248}{5}$, $x_{0}=\frac{208}{1539}, y_{-1}=\frac{287793}{3328}, y_{0}=\frac{5}{112}$.

\section{REFERENCES}

[1] Agarwal RP, Wong PJ. Advanced topics in difference equations (Vol. 404). Springer Science \& Business Media, 2013.

[2] Camouzis E, Ladas G. Dynamics of third order rational difference equations with open problems and conjectures, volume 5 of Advances in Discrete Mathematics and Applications, Chapman \& Hall/CRC, Boca Raton, FL; 2008.

[3] Camouzis E, Papaschinopoulos G. Global asymptotic behavior of positive solutions on the system of rational difference equations $\mathrm{xn}+1=1+$ xnyn- $\mathrm{m}$, yn $+1=1+$ ynxn- m. Appl. Math. Lett. 2004; 17 (6): pp. 733-737.

[4] Din Q, Qureshi MN, Khan AQ. Dynamics of a fourth-order system of rational difference equations. Adv. Difference Equ. 2012; 2012:215, pp. 1-15.

[5] Elaydi S. An Introduction to Difference Equations. Springer-Verlag, 1996.

[6] Gelisken A, Kara M. Some general systems of rational difference equations. Journal of Difference Equations 2015; 2015, pp. 1-7. 


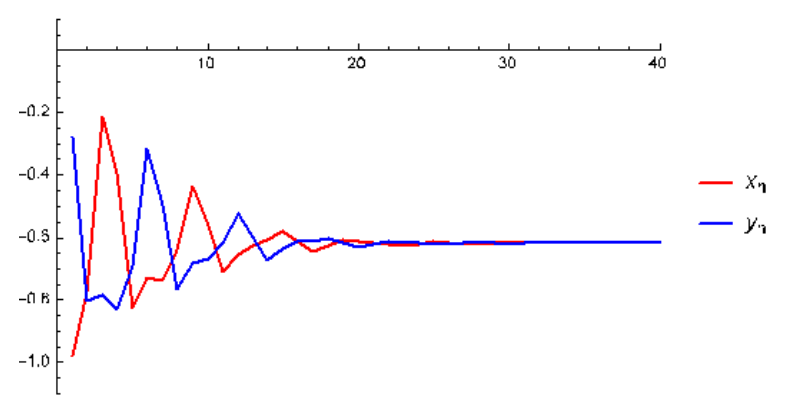

Figure 6. Plot of system (1.1) for initial values $x_{-1}=-0.1$, $x_{0}=-0.8, y_{-1}=-0.9, y_{0}=-0.2$.

[7] Göcen M, Güneysu M. The global attractivity of some rational difference equations. J. Comput. Anal. Appl. 2018; 25 (7), pp. 1233-1243.

[8] Göcen M, Cebeci A. On the Periodic Solutions of Some Systems of Higher Order Difference Equations. Rocky Mountain J. Math. 2018; 48 (3), pp. 845-858.

[9] Grove EA, Ladas G. Periodicities in nonlinear difference equations. Chapman and Hall/CRC, 2004.

[10] Kent CM, Kosmala W, Radin MA, Stević S. Solutions of the difference equation $x_{n+1}=$ $x_{n} x_{n-1}-1$. Abstr. Appl. Anal. 2010; 2010, pp. 1-13.

[11] Kent CM, Kosmala W. On the Nature of Solutions of the Difference Equation $x_{n+1}=$ $x_{n} x_{n-3}-1$. International Journal of Nonlinear Analysis and Applications 2011; 2 (2), pp. $24-43$.

[12] Kent CM, Kosmala W, Stević S. Long-term behavior of solutions of the difference equation $x_{n+1}=x_{n-1} x_{n-2}-1$. Abstr. Appl. Anal. 2010; 2010, pp. 1-17.

[13] Kent CM, Kosmala W, Stević S. On the difference equation $x_{n+1}=x_{n} x_{n-2}-1$. Abstr. Appl. Anal. 2011; 2011, pp. 1-15.

[14] Kocic VL, Ladas G. Global behavior of nonlinear difference equations of higher order with applications. Vol. 256. Springer Science \& Business Media, 1993.

[15] Kulenovic MR, Ladas G. Dynamics of second order rational difference equations: with open problems and conjectures. Chapman and Hall/CRC, 2001.

[16] Kulenovic MR, Nurkanovic M. Asymptotic behavior of a system of linear fractional difference equations. J. Inequal. Appl. 2005; 2005 (2), pp. 1-17.

[17] Kurbanlı AS, Çinar C, Yalçinkaya İ. On the behavior of positive solutions of the system of rational difference equations $\mathrm{xn}+1=\mathrm{xn}-1 \mathrm{ynxn}-1+1$, yn $+1=\mathrm{yn}-1 \mathrm{xnyn}-1+1$. Mathematical and Computer Modelling 2011; 53 (5-6), pp. 1261-1267.

[18] Liu K, Li P, Han F, Zhong W. Behavior of the Difference Equations x n+ 1= x n x n-1-1. J. Comput. Anal. Appl. 2017; 201722 (7), pp. 1361-1370.

[19] Okumuş İ, Soykan Y. On the Stability of a Nonlinear Difference Equation. Asian Journal of Mathematics and Computer Research 2017; 17 (2), pp. 88-110.

[20] Okumuş İ, Soykan Y. Some Technique To Show The Boundedness Of Rational Difference Equations. Journal of Progressive Research in Mathematics 2018; 13 (2), pp. 2246-2258.

[21] Okumuş İ, Soykan Y. Dynamical behavior of a system of three-dimensional nonlinear difference equations. Adv. Difference Equ. 2018; 2018:224, pp. 1-15.

[22] Papaschinopoulos G, Schinas C.J. On a system of two nonlinear difference equations. J. Math. Anal. Appl. 1998; 219 (2), pp. 415-426.

[23] Stevic S, Alghamdi MA, Maturi DA, Shahzad N. On the Periodicity of Some Classes of Systems of Nonlinear Difference Equations. Abstr. Appl. Anal. 2014; 2014, pp. 1-6.

[24] Stevic S. On the difference equation $x_{n+1}=\alpha+x_{n-1} / x_{n}$. Comput. Math. Appl. 2008; 56, pp. 1159-1171.

[25] Stevic S. On some solvable systems of difference equations. Appl. Math. Comput. 2012; 218, pp. 5010-5018.

[26] Taşdemir E, Soykan Y. On the Periodicies of the Difference Equation $x_{n+1}=x_{n} x_{n-1}+\alpha$. Karaelmas Science and Engineering Journal 2016; 6 (2), pp. 329-333. 
[27] Taşdemir E, Soykan Y. Long-Term Behavior of Solutions of the Non-Linear Difference Equation $x_{n+1}=x_{n-1} x_{n-3}-1$. Gen. Math. Notes 2017; 38 (1), pp. 13-31.

[28] Taşdemir E, Soykan Y. Stability of Negative Equilibrium of a Non-Linear Difference Equation. J. Math. Sci. Adv. Appl. 2018; 49 (1), pp. 51-57.

[29] Taşdemir E, Soykan Y. Dynamical Analysis of a Non-Linear Difference Equation. J. Comput. Anal. Appl. 2019; 26 (2), pp. 288-301.

[30] Wang Y, Luo Y, Lu Z. Convergence of solutions of $x_{n+1}=x_{n} x_{n-1}-1$. Appl. Math. E-Notes 2012; 12, pp. 153-157. 